\title{
Redes de conhecimento na comunicação científica em âmbito formal: panorama via Biblioteca Brasileira de Teses e Dissertações (2008-2018)
}

\section{Knowledge networks in the scientific communication in a formal field: panorama of Biblioteca Brasileira de Teses e Dissertações (2008-2018)}

\author{
Ketlen Stueber ${ }^{1}$, Maria do Rocio Fontoura Teixeira ${ }^{2}$ \\ ${ }^{1}$ Universidade Federal do Rio Grande do Sul: Porto Alegre, Rio Grande do Sul, Brasil. ORCID: https://orcid.org/0000-0002-2171-0365 \\ 2 Universidade Federal do Rio Grande do Sul: Porto Alegre, Rio Grande do Sul, Brasil. ORCID: https://orcid.org/0000-0002-9888-7185
}

Autor para correspondência/Mail to: Ketlen Stueber, ketistueber@hotmail.com

Copyright (c) 2019 Stueber\& Teixeira. Todo o conteúdo da Revista (incluindo-se instruções, política editorial e modelos) está sob uma licença Creative Commons Atribuição-NãoComercial-Compartilhalgual 3.0 Não Adaptada. Ao serem publicados por esta Revista, os artigos são de livre uso em ambientes educacionais, de pesquisa e não comerciais, com atribuição de autoria obrigatória. Mais informações em http://revistas.ufpr.br/atoz/about/submissions\#copyrightNotice.

\begin{abstract}
Resumo
Introdução: Apresenta o estado da arte de pesquisas de pós-graduação sobre redes de conhecimento na produção da comunicação científica nos últimos dez anos (2008-2018). Método: pesquisa qualitativa, utiliza como instrumento de coleta de dados a busca simples pelo termo "redes de conhecimento" na Biblioteca Brasileira de Teses e Dissertações do Instituto Brasileiro de Informação em Ciência e Tecnologia. Resultados: 0 critério norteador para filtrar a busca foi selecionar pesquisas sobre redes de conhecimento estabelecidas a partir do conhecimento produzido por instituições e grupos de pesquisas, disponibilizadas em periódicos científicos, diretórios, anais de eventos, livros e outras publicações produzidas entre pares. A investigação dos estudos encontrados efetivou-se a partir de pesquisa bibliográfica. Foram encontrados sete estudos, sendo quatro dissertações e três teses. Conclusões: conclui-se que as pesquisas abordam a produção do conhecimento científico em diferentes objetos, seja na esfera de concepção com base em grupos de estudo e linhas de pesquisa, seja na esfera da documentação com o estudo sobre artigos, bases de dados e periódicos científicos ou em ambos através dos diretórios de pesquisa.
\end{abstract}

Palavras-chave: Redes de conhecimento; Produção do conhecimento científico; Instituições de pesquisa.

\begin{abstract}
Introduction: It presents the state of the art of graduate research on knowledge networks in the development of scholarly communication in the last ten years (2008-2018). Methods: It uses qualitative research and it collects the data searching for the keyword "knowledge networks" using the simple search in the Brazilian Library of Theses and Dissertations of the Brazilian Institute of Information in Science and Technology. Results: The criterion for filtering the search was to select research on knowledge networks established from the knowledge produced by institutions and research groups, available in scientific journals, directories, annals of events, books, and other publications produced among peers. The investigation of the studies used bibliographic research. It founds seven studies, four dissertations, and three theses. Conclusions: It concludes that the researches approach the production of scientific knowledge in different objects, either in the sphere of conception based on study groups and lines of research, or in the sphere of documentation with the study of articles, databases, and scientific journals or both through the search directories.
\end{abstract}

Keywords: Networks of knowledge; Production of scientific knowledge; Research institutions.

\section{INTRODUÇÃO}

Imagine uma grande teia com gotas de orvalho. Cada gotícula é um ponto que se interliga com outros pontos por meio dos finos fios que a compõem. A analogia da teia de aranha é uma das formas mais comuns de representação do conhecimento. Seja de modo abstrato ou real, a complexidade existente acerca do assunto é evidente. O conhecimento é dialógico e, quando comparado a uma grande "teia" ou rede, se transforma num sistema vivo, intenso, transversal e interdisciplinar. Várias trocas são estabelecidas nesta cadeia que pode ser acessada de diferentes pontos, todos compostos por informações e inscrições.

Este artigo pertence a um conjunto de estudos voltados a compreender as redes de conhecimento estabelecidas a partir do primeiro periódico brasileiro de zoologia. A revista Iheringia, Série Zoologia, criada em 1957 pelo Museu de Ciências Naturais do Rio Grande do Sul, é reconhecida em âmbito nacional e internacional em seu campo. Nesse contexto, o estudo das redes de conhecimento científico produzido por instituições de pesquisa, diretórios e grupos de estudos, registrado em diferentes suportes, é altamente relevante. Torna-se imprescindível questionar: Qual o panorama da pesquisa brasileira sobre redes de conhecimento na produção da comunicação científica em âmbito formal (produzida entre pares)?

As respostas para essa questão contribuem para estabelecer o estado da arte da pesquisa a partir das redes de conhecimento, enquanto base epistemológica, e a comunicação científica formal, enquanto objeto de estudo (em nível nacional de mestrado e doutorado). Este artigo mapeia pesquisas de pós-graduação disponibilizadas pela Biblioteca Brasileira de Teses e Dissertações (BBTD) do Instituto Brasileiro de Informação em Ciência e Tecnologia (IBICT). O estudo de abordagem qualitativa com base em pesquisa bibliográfica se estabelece dentro de um recorte temporal de dez anos (2008-2018). 


\section{A COMPOSIÇÃO DOS PONTOS QUE CONECTAM AS REDES: INFORMAÇÕES E INSCRI- ÇÕES}

O conceito de informação na contemporaneidade desafia a perspectiva unívoca e positivista, principalmente se fundamentada sob premissas epistemológicas e empíricas da sociologia do conhecimento. A informação é, acima de tudo, um fenômeno humano. "[...] Informação é o que é o que é informativo para uma determinada pessoa. O que é informativo depende das necessidades interpretativas de habilidades do indivíduo (embora estas sejam frequentemente compartilhadas em uma mesma comunidade de discurso)" (Capurro \& Hjørland, 2007, p.155). Percebe-se que o conceito de informação apresenta, pela perspectiva dos autores, características humanísticas e interdisciplinares.

Tal conceito necessita ser estudado, conforme afirmam Capurro e Hjørland (2007, p.176), por meio da perspectiva do campo em que está inserido: "o que distingue as diferentes teorias da informação é, portanto, não apenas o conceito de informação em si. É, em um grau mais elevado, a natureza do mecanismo de liberação (ou mecanismos de processamento de informação), os seletores ou intérpretes". Segundo os autores, a informação pode ser considerada desde os seus aspectos físicos e tecnológicos, lógicos e matemáticos, linguísticos e semânticos (vocabulários controlados, teorias discursivas e de significações). O importante é evitar sobrepor e hierarquizar suas perspectivas, pois isso gera limitações e empobrecimento epistêmico ao campo da Ciência da Informação.

As discussões acerca do conceito de informação implicam questões epistemológicas e paradigmáticas. Capurro (2003) aponta três paradigmas principais referentes ao conceito: paradigma físico; paradigma cognitivo e paradigma social. O paradigma físico trata da informação e seus suportes tangíveis (objetos, livros, documentos, computadores, celulares, entre outros). O paradigma cognitivo busca discutir o conceito de informação a partir de redes mentais formadas por meio da aquisição e troca de conhecimento em âmbito intelectual. Esse paradigma questiona de que forma os processos informacionais podem compor ou não redes mentais a partir da aquisição de informações efetivas que constituem os processos de saber. Já o paradigma social discute como se dá a interação dos indivíduos que compartilham e socializam informações efetivando-as (ou não) em conhecimento. O autor entende que a informação em sua dimensão epistemológica (Ciência da Informação), não deve isolar seus paradigmas, mas sim pensá-los em conjunto.

Segundo Mc Garry (1999, p.11), "a informação deve ser representada para nós de alguma forma e transmitida por algum tipo de canal". Sinais, signos e símbolos são os três principais veículos para a transmissão de informações, mas é por meio da cultura e da significação que esses três elementos se concretizam. Desse modo, a informação é conhecimento potencial arraigado ao fator humano. Para Mc Garry (1999, p.31) nela contém "[...] um potencial de ação, como a eletricidade à espera da mão humana que toque no interruptor. Uma visão mecanicista extremada deixaria os seres humanos na condição de epifenômenos, meras frivolidades, reflexos passivos de uma realidade material a propósito ou plano". O autor defende ainda que não se deve tratar a informação sem as pessoas e as pessoas sem a informação, pois informação é conhecimento potencial. É preciso legitimar suas formas denotativas e conotativas, ou seja, considerar também os enunciados, seus produtores e receptores.

As reflexões sobre a dialogicidade entre o campo da Informação, Comunicação e Ciências Sociais, elaborados por González de Gómez (2012) complementam a discussão ao agregar a vertente habermasiana de Ação comunicativa e ressignificá-la. Assim, a ação da informação é considerada como:

[...] aquela realizada por atores sociais em suas práticas e atividades, ancoradas culturalmente numa forma de vida e geradas em comunidades epistêmicas ou configurações coletivas de relações intersubjetivas. No contexto sociocultural, uma ação de informação poderá orientar-se preferencialmente em direção a processos de objetivação (uso representativo da linguagem) ou oferecer garantias performáticas à busca de entendimento mútuo (uso comunicativo da linguagem)(González de Gómez, 2012, p.27).

Ao que denomina Ação de informação, a autora propõe três estratos principais: semântico pragmático; dispositivo operacional e tecnológico e; regulatório. Esses estratos, efetivados pela Ação da Informação, relacionam-se intrinsecamente com os níveis e formas de integração social, por meio da cultura, da potência do imaginário coletivo e de sua produção e reprodução. Para Latour (2006, p.22), a informação é

[...] uma relação estabelecida entre dois lugares, o primeiro, que se torna uma periferia, e o segundo, que se torna um centro, sob a condição de que entre os dois circule um veículo que denominamos muitas vezes forma, mas que, para insistir em seu aspecto material, eu chamo de inscrição.

Nessa perspectiva, a informação é entendida enquanto fenômeno gerador de movimento e dinamicidade e estabelece-se a partir de uma dimensão epistêmica que, sem a necessidade de matéria, cria a forma de distintos objetos e fenômenos por meio de descrições. É esta ação que permite que o pesquisador, cientista, artista ou qualquer outro ator social transforme/represente a matéria em inscrição. Entende-se por inscrição:

Termo geral referente a todos os tipos de transformação que materializam uma entidade num signo, num arquivo, num documento, num pedaço de papel, num traço. Usualmente, mas nem sempre as inscrições são bidimensionais, 
sujeitas a superposição e combinação. São sempre móveis, isto é, permitem novas translações e articulações ao mesmo tempo que mantém intactas algumas formas de relação (Latour, 2017, p.362).

A partir da ação de transformar matéria em informação e informação em inscrição e relacioná-las, aproximando objetos e fenômenos que possuem relação ou algum tipo de proximidade dentro de dimensões interdisciplinares, Latour (2000, 2006, 2017) elabora a teoria das redes. De acordo com seus estudos, as redes são estruturas por onde circula e se transforma o conhecimento. Os temas ou fenômenos que compõem as redes estão interligados (conforme seu grau de compatibilidade) em movimentos aleatórios e não hierárquicos.

Para Latour (2006) as redes estão imersas de relatividade e os fenômenos se deslocam o tempo todo do centro para a periferia e vice-versa. Mesmo composta por um "núcleo" (geralmente composto por conhecimento estático) e sistemas periféricos (em que o conhecimento muda de status com mais facilidade), Latour (2017) afirma que o conhecimento produzido nas e pelas redes permite constantemente translações e articulações. As translações remetem aos deslocamentos das informações e inscrições nas redes. A veracidade dos fenômenos está na forma que os mesmos se mantêm e circulam. Seus movimentos é que lhes garantem validade. Quem rege o movimento das redes são os atores sociais ligados a ela com base nas inscrições e nos métodos utilizados para compreender e impulsionar tais fenômenos.

\section{A COMUNICAÇÃO CIENTÍFICA: ESPAÇO PRIMORDIAL DAS REDES DE CONHECIMENTO}

"Redes de conhecimento" e "comunicação científica" são conceitos amplos e carregam em si distintos sentidos e significados. Em épocas em que o conhecimento produzido em universidades e centros de pesquisas não eram disponibilizados na internet, mas através de correspondências e telefonemas, a troca de ideias, as descobertas e as pesquisas evoluíam em outro ritmo. Ao considerar as redes enquanto meios de colaboração e dialogicidade entre fenômenos e objetos, pode-se dizer que o estabelecimento de redes de conhecimento permeia a ciência desde antes da era da informação trazida pelas novas tecnologias.

Foi nesse contexto que Thomas Kuhn buscou compreender o fenômeno científico. Para isso, além da interação com seus pares, Kuhn (2007) procurou entender a ciência mesclando as perspectivas científicas da física e áreas afins com a filosofia e as ciências sociais. Ao analisar a produção científica não apenas em livros, mas em artigos de periódicos e anais de eventos, Kuhn (2007) contribuiu para as ciências com as discussões acerca do conceito de paradigmas científicos. Deste modo, a produção científica registrada e formalizada em periódicos e outros documentos oficiais da academia são relevantes objetos de estudo para compreender a ciência e sua dinamicidade.

O termo comunicação científica pode referenciar diversos sentidos. Caribé (2015) afirma que essa expressão é genérica, pois a partir dela ressurgem outros vocábulos associados e/ou subordinados como, por exemplo, popularização da ciência, difusão, disseminação ou divulgação científica. Segundo a autora, esses termos se referem aos processos de produção científica, criados a partir de pesquisadores e instituições para levar conhecimentos a diversos grupos sociais. No âmbito de recepção destes conhecimentos, os termos compreensão pública da ciência, educação científica e alfabetização científica não são processos, mas sim habilidades/competências adquiridas pelo público (Caribé, 2015).

A partir da produção de vários pensadores que se dedicaram ao tema, Caribé (2015), afirma que a comunicação científica perpassa por pelo menos três esferas: concepção, documentação e popularização. Na esfera da concepção, a troca de conhecimentos se dá de modo informal entre os pares, em sua maioria pertencentes a grupos sociais e culturais homogêneos. Grande quantidade de informações, vocabulários extensos e especializados estão presentes nas reuniões informais entre os grupos de pesquisa. Na esfera da documentação, grande volume de informações científicas, métodos e discursos são compartilhados, mas a quantidade de interações sociais é pequena. Nesta etapa a comunicação se dá por canais formais que produzem registros e documentos fundamentados em estudos e pesquisas. Durante este estágio, o estilo e a linguagem podem ser fatores limitantes para a compreensão de grupos que não integram o mesmo campo.

Na esfera da popularização, o conhecimento chega ao público por meio de diversos processos de mediação em audiências de massa: jornais, revistas, programas de televisão, internet (vídeos curtos e postagens nas redes sociais). As ideias científicas se incorporam ao discurso público sem haver necessariamente um domínio crítico e aprofundado do conhecimento, tampouco dos usos e vocabulários especializados. Além dos efeitos de popularização para o público em geral, os temas ocasionam simultaneamente debates na academia e grupos de pesquisas, fomentando assim a elaboração de novos conhecimentos. Percebe-se que as esferas da comunicação científica estão interligadas e suas fases se dão de modo natural e subsequente sem que hajam interrupções.

\section{PROCEDIMENTOS METODOLÓGICOS}

A qualificação da abordagem de uma pesquisa se dá a partir dos objetivos delineados pelo pesquisador em relação aos resultados que busca em seu estudo, podendo esses serem de ordem qualitativa, quantitativa ou ambos. A pesquisa de abordagem qualitativa possui como principais objetivos a descrição, a compreensão e a interpretação 
dos fatos. A validação dos dados ocorre pela consistência obtida no exame de elementos teóricos e os achados da investigação. De acordo com Martins e Theóphilo (2007) são dados qualitativos: descrições, citações diretas de pessoas, documentos, gravações de entrevistas, interações entre indivíduos.

Na pesquisa qualitativa, o significado torna-se o conceito central da investigação (Minayo, 2001). Mesmo que determinado estudo apresente dados numéricos, a abordagem qualitativa pode utilizar-se dos mesmos para justificar a importância dos conteúdos argumentativos que busca compreender e elaborar. Para Minayo (2001, p.24) os autores que seguem a corrente qualitativa não se preocupam em quantificar, mas sim conhecer e explicar fenômenos e dinâmicas sociais, "a dialética pensa a relação da quantidade como uma das qualidades dos fatos e fenômenos". Portanto, o presente estudo insere-se numa pesquisa de abordagem qualitativa.

Corpus da pesquisa: Concebida em 2002, a BDTD do IBICT disponibiliza, em acesso aberto e gratuito, as pesquisas defendidas nas instituições brasileiras de ensino e pesquisa. Dentre suas principais linhas de atuação destaca-se o intuito de desenvolver um sistema de biblioteca eletrônica em nível nacional para divulgar pesquisas de teses e dissertações de todas as instituições de ensino brasileiras, inclusive aquelas que não possuem sistemas automatizados. É mantida pela Financiadora de Estudos e Pesquisas (FINEP) e sua criação contou com a participação de representantes de vários órgãos: Conselho Nacional de Desenvolvimento Científico e Tecnológico (CNPq), Coordenação de Aperfeiçoamento de Pessoal de Nível Superior (CAPES) e Secretaria de Educação Superior (SESu), Universidade de São Paulo (USP), Pontifícia Universidade Católica do Rio de Janeiro (PUCRIO) e a Universidade Federal de Santa Catarina (UFSC). Desde sua criação, a BDTD passou por diversas fases de renovação tecnológica nos sistemas de metadados, coleta e interface do portal. O último registro de atualização aconteceu em 2016/2017 (Instituto Brasileiro de Informação em Ciência e Tecnologia (IBICT)., [2018]).

Coleta: a partir da técnica de levantamento Bibliográfico (Gil, 2008), a pesquisa utiliza como instrumento de coleta de dados a busca simples pelo termo "redes de conhecimento" na BBTD do Instituto Brasileiro de Informação em Ciência e Tecnologia IBICT, considerando como intervalo o recorte temporal de dez anos (2008-2018). A busca simples pelo termo "redes de conhecimento" deu-se de modo proposital com o intuito de alcançar quantidade ampla de resultados.

Foram recuperadas 123 pesquisas de 37 instituições, sendo 74 dissertações e 50 teses. O critério norteador para filtrar a busca foi selecionar pesquisas sobre redes de conhecimento estabelecidas a partir do conhecimento produzido por instituições e grupos de pesquisas, disponibilizado em periódicos científicos, diretórios, anais de eventos, livros e outras publicações produzidas entre pares. A investigação dos estudos encontrados efetivou-se a partir de pesquisa bibliográfica (Gil, 2008).

Análise: Dentre os principais procedimentos para análise e descrição dos dados na abordagem qualitativa, encontram-se: análises de conteúdo, de discurso, ou análise dialética (Minayo, 2001). A análise de conteúdo (AC) é formada por um conjunto de técnicas de análise e procedimentos objetivos e sistemáticos de descrição de informações em busca de desvelar os sentidos no plano de análise. É utilizada para a identificação e sistematização na coleta e análise dos dados organizada em três etapas principais, segundo Bardin (2009). A pré-análise fundamenta-se na seleção, delimitação do objeto e aplicação de regras de recorte e delimitação. A exploração do material consiste na administração das técnicas sobre o corpus. O tratamento dos resultados e interpretações pode ser desenvolvida através de operações estatísticas (frequência do uso dos termos), síntese e seleção dos resultados (inferências; interpretações, orientações para uma nova análise) e/ou pela utilização dos resultados para fins teóricos ou pragmáticos.

Bauer (2013) caracteriza diferentes suportes de registros em que a análise de conteúdo pode ser aplicada:

a) unidades físicas (livros, cartas, filmes e vídeos em geral);

b) unidades proposicionais: Presente nos discursos de sujeitos e grupos sociais, as unidades proposicionais são núcleos lógicos de frases em que "proposições complexas são desconstruídas em núcleos na forma de sujeito/verbo/objeto" (Bauer, 2013, p.198); e

c) unidades temáticas ou semânticas: enunciados e textos.

\section{RESULTADOS: APRESENTAÇÃO E DISCUSSÃO}

A coleta de dados aconteceu em abril de 2019, através da inserção do termo "redes de conhecimento" no campo de busca da BBTD do IBICT, considerando o intervalo de tempo de dez anos (2008-2018). No total, foram encontrados 123 resultados, 74 dissertações e 50 teses oriundas de 37 instituições. O 1 a seguir apresenta um ranking das cinco primeiras universidades que pesquisam o tema. 


\begin{tabular}{|l|c|}
\hline Instituição & Número de Pesquisas \\
\hline Universidade Federal de Santa Catarina (UFSC) & 16 \\
Universidade de São Paulo (USP) & 13 \\
Universidade Federal de Pernambuco (UFPB) & 12 \\
Universidade Estadual do Rio de Janeiro (UERJ) & 07 \\
Universidade Federal do Rio Grande do Sul (UFRGS) & 07 \\
\hline
\end{tabular}

Quadro 1. Ranking das universidades que pesquisam sobre redes de conhecimento conforme estudos publicados na BBTD do IBICT (20082018)

Fonte: Elaborado pelas autoras (2019).

Dentre as cinco instituições que apresentam pesquisas mais recorrentes sobre o tema, todas são públicas, três de caráter federal e duas estaduais. A frequência de termos associados ao tema redes de conhecimento, presentes no montante das 123 pesquisas foram: Gestão do conhecimento (17); Conhecimento (12); Redes Sociais (10); Análise de Redes Sociais (7); Redes (5); Comunidades de Prática (4).

Para refinar a busca de acordo com os critérios de seleção deste estudo, foram elencados trabalhos que abordassem as redes de conhecimento na produção da comunicação científica em âmbito formal (produzida entre pares). Conforme a perspectiva teórica fundamentada neste estudo, consideraram-se pesquisas que abordassem o processo de produção do conhecimento na esfera da concepção e registro, processos que antecedem a divulgação e o conhecimento científico na esfera da popularização (Caribé, 2015).

Portanto, teses e dissertações sobre a colaboração entre pesquisadores de centros de pesquisa (universidades, museus e demais instituições), grupos de pesquisas e produção em fontes documentais (periódicos nacionais e internacionais, apresentações ou publicações em congressos, anais, capítulos de livros, livros, manuais ou relatório técnico, monografias) foram selecionadas. Sete pesquisas foram encontradas (Quadro 2) e estão apresentadas em ordem temporal.

\begin{tabular}{|c|c|c|c|}
\hline Autor(ano) & Título & $\begin{array}{l}\text { Natureza } \\
\text { do Estudo }\end{array}$ & Universidade \\
\hline Lima, M. (2009) & $\begin{array}{l}\text { Redes de co-autoria científica no Programa de Pós- } \\
\text { Graduação em Geociências da UFRGS }\end{array}$ & Dissertação & UFRGS \\
\hline Felix (2011) & $\begin{array}{l}\text { A pesquisa nos grupos de pesquisa: cibernética peda- } \\
\text { gógica - laboratório de linguagens digitais - LLD }\end{array}$ & Dissertação & USP \\
\hline Lima, T. (2012) & $\begin{array}{l}\text { Produção do conhecimento científico brasileiro = o } \\
\text { caso de uma instituição de pesquisas em Campinas }\end{array}$ & Tese & UNICAMP \\
\hline Freitas (2013) & $\begin{array}{l}\text { Diretório dos grupos de pesquisa do } \mathrm{CNPq} \text { - como } \\
\text { ferramenta de análise de redes: um estudo de caso do } \\
\mathrm{CDS} / \text { UnB }\end{array}$ & Tese & UFBA \\
\hline Alvarenga (2014) & $\begin{array}{l}\text { Redes de co-autoria científica no Programa de Pós- } \\
\text { Graduação em Geociências da UFRGS }\end{array}$ & Dissertação & UnB \\
\hline Costa (2016) & $\begin{array}{l}\text { Rede de pesquisa no Instituto de Pesquisa Econômica } \\
\text { Aplicada (IPEA): uma análise da produção cientifica a } \\
\text { partir dos Textos para Discussão (TD) }\end{array}$ & Dissertação & UnB \\
\hline Zattar (2017) & $\begin{array}{l}\text { Prática informacional em redes no domínio da gover- } \\
\text { nança da água: um estudo sobre o processo de produ- } \\
\text { ção do conhecimento }\end{array}$ & Tese & UFRJ \\
\hline
\end{tabular}

Quadro 2. Pesquisas sobre redes de conhecimento na produção da comunicação científica em teses e dissertações (2008-2018) Fonte: Elaborado pelas autoras (2019).

No total, quatro dissertações e três teses compõem os estudos selecionados. Dentre os autores, seis são do sexo feminino e um masculino. O campo de redes do conhecimento na comunicação científica, muito além de sua característica híbrida, está composto de interdisciplinaridade, pois, as pesquisas são oriundas de diferentes áreas do conhecimento:

a) Programa de Pós-graduação em Sociologia da UFRGS (Alvarenga, 2009);

b) Programa de Pós-graduação em Ciências da Comunicação da Escola de Comunicação e Artes da Universidade de São Paulo USP (Costa, 2011);

c) Programa de Doutorado em Ciências Sociais, Universidade Estadual de Campinas UNICAMP (Alvarenga, 2012);

d) Programa de Doutorado em Administração, Universidade Federal da Bahia UFBA (Alvarenga, 2013); 
e) Programa de Pós-Graduação em Política e Gestão de Ciência e Tecnologia submetida ao Centro de Desenvolvimento Sustentável da Universidade de Brasília - CDS/UnB (Alvarenga, 2014);

f) Departamento de Economia da Universidade de Brasília (UnB) (Costa, 2016); e

g) Programa de Pós-Graduação em Ciência da Informação, convênio entre o Instituto Brasileiro de Informação em Ciência e Tecnologia e a Universidade Federal do Rio de Janeiro UFRJ/Escola de Comunicação (Zattar, 2017).

A Figura 1 retrata o posicionamento das pesquisas a partir das esferas de comunicação formal: concepção, documentação e ambas (Caribé, 2015).

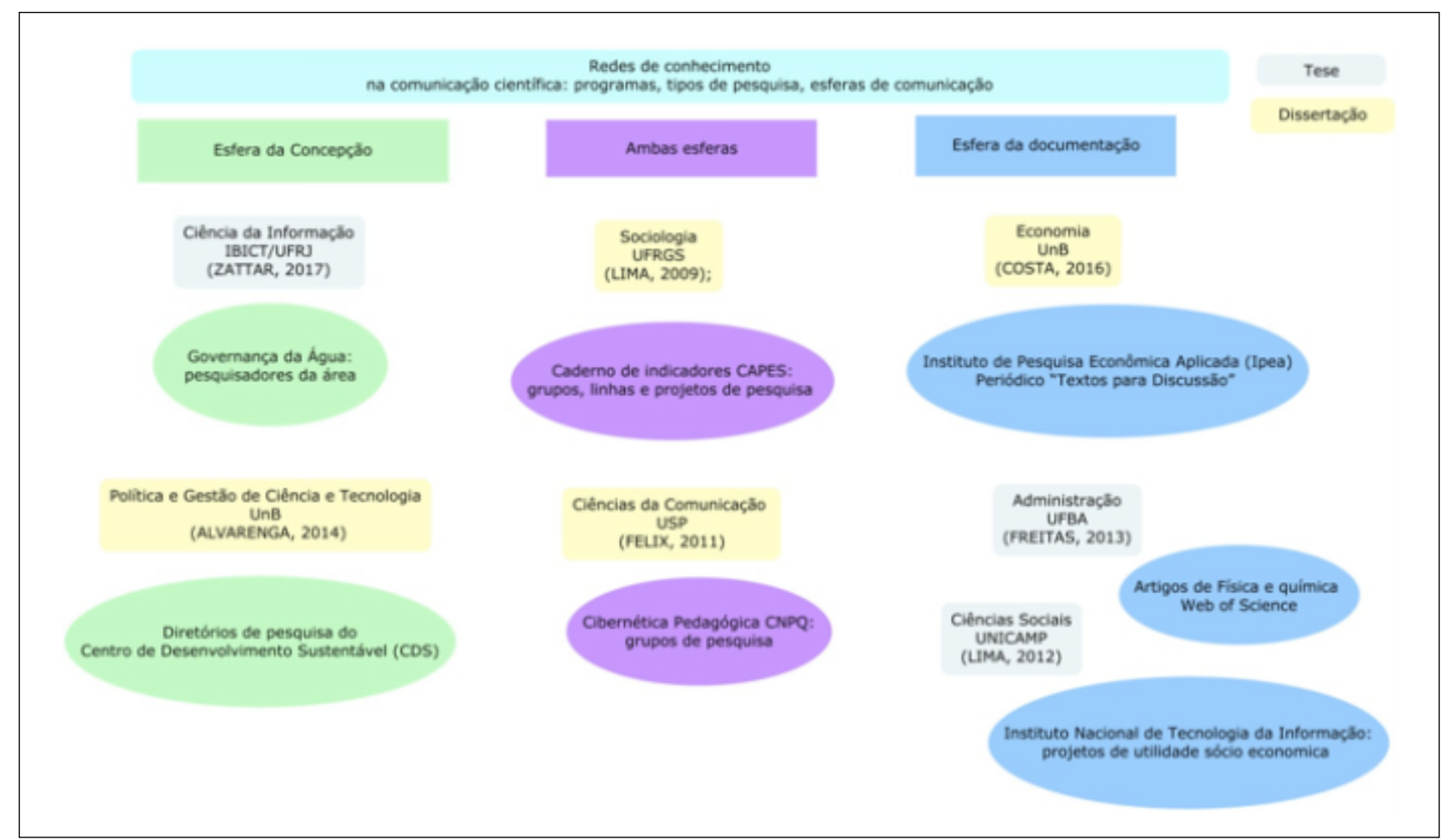

Figura 1. Esferas de comunicação das teses e dissertações sobre redes de conhecimento na comunicação científica na BBTD do IBICT (20082018)

Fonte: Elaborado pelas autoras (2019).

A esfera da concepção estuda as redes de conhecimento formadas entre pesquisadores que se concentram em estudar a gestão das águas (Zattar, 2017) e diretórios de pesquisa sobre desenvolvimento sustentável (Alvarenga, 2014). A esfera da documentação fundamenta suas redes a partir de artigos científicos (Alvarenga, 2013; Costa, 2016) e projetos de aplicação socioeconômica (Alvarenga, 2012). A verificação de redes com base em grupos de pesquisa (Alvarenga, 2009; Costa, 2011) promove conhecimentos dialógicos entre os processos formais e de concepção, deste modo pode ser considerado em ambas as esferas. A descrição mais detalhada das pesquisas segue a sequência temporal em que foram defendidos (2009-2017).

O estudo de redes sociais é considerado potencial gerador de subsídios para a gestão de ciência e tecnologia para Alvarenga (2009). Com base na escola bourdiana, o autor parte da Análise de Redes Sociais (ARS) para identificar e caracterizar as redes de coautoria científica do programa de pós-graduação em Geociências da UFRGS. O corpus estudado foi o Caderno de Indicadores da CAPES com foco nos grupos, linhas e projetos de pesquisa de 1998 até 2006. No período de 1998 a 2000 o número de atores identificados é de 524 em 11.596 laços de coautoria, enquanto em 2004 a 2006, o resultado sobre para 741 atores em 14.188 laços. Alvarenga (2009) conclui que a evolução estrutural das redes de coautoria é afetada, em maior parte, pelas linhas e projetos de pesquisa e em menor parte pelos grupos de pesquisa.

Comunicação e informação, ciência, tecnologia e educação são os pontos que interligam a rede de conhecimentos tecida no estudo de Costa (2011). Além da criação e descrição de uma página oficial para o grupo de pesquisa Cibernética Pedagógica na plataforma do CNPQ, a autora apresenta outros 17 grupos de pesquisa pertencentes ao mesmo campo.

A análise da produção científica em instituições públicas é o foco do estudo de Alvarenga (2012). Em sua tese, a pesquisadora retrata as dinâmicas de pesquisa e produção do Centro de Tecnologia da Informação Renato Archer (CTI) transformado em 2000 no Instituto Nacional de Tecnologia da Informação. A partir de projetos de utilidade socioeconômica, o conhecimento científico torna-se o núcleo de uma rede criada por pontos de saberes 
interconectados, considerando as mediações das políticas nacionais de ciência e tecnologia em diferentes períodos no Brasil. Por meio de pesquisa etnográfica e entrevistas, Alvarenga (2012) também apresenta a importância da Lei da Inovação e os padrões de relacionamento estabelecido entre os pesquisadores.

A socialização do conhecimento, aliada às subjetividades dos pesquisadores e à formação de redes de colaboração, compõe os elementos centrais para o estudo de Alvarenga (2013). Para a autora, a combinação destes fatores pode influir de maneira positiva nos resultados de produção científica. A partir de 898 artigos disponíveis na Web of Science, produzidos por 63 pesquisadores de pós-graduação da área de química e física da Universidade Federal da Bahia (UFBA), no período de 1998 a 2009, Alvarenga (2013) analisa o comportamento das redes de colaboração dos respectivos pesquisadores e a formação de capital social dos grupos por eles formados sem desconsiderar o comportamento individual e subjetivo dos mesmos.

A informação produzida nos diretórios de Grupos de Pesquisa foi o objeto de estudo de Alvarenga (2014). Para isto, a pesquisadora identificou as redes de pesquisa existentes no Centro de Desenvolvimento Sustentável (CDS) da Universidade de Brasília (UnB) e buscou saber da comunidade científica integrante de que forma se apropriam deste recurso. Para Alvarenga (2014), o diretório possui um grande potencial de auxílio, produção de pesquisa e conhecimento.

O Instituto de Pesquisa Econômica Aplicada (Ipea) é uma fundação pública vinculada ao Ministério do Planejamento e a produção científica ali desenvolvida é analisada por Costa (2016). O Ipea foi criado com o objetivo de apoiar o Governo Federal na implementação de políticas públicas por meio de atividades de pesquisa e planejamento (Costa, 2016). A autora constrói as redes de conhecimento com base na ARS para compreender as redes de colaboração e coautoria de 37 anos de publicação científica da série periódica "Textos para Discussão". Costa (2016) conclui que o periódico contribui na construção do conhecimento científico brasileiro pelos elos criados entre os pesquisadores em âmbito nacional e internacional e por proporcionar o acesso aberto às pesquisas publicadas pela revista no campo da economia e administração pública.

A gestão das águas enquanto área do conhecimento é denominada Governança da Água. Zattar (2017) identifica as configurações das redes de pesquisadores pertencentes a este campo científico. A autora emprega os conceitos de rede social e prática informacional como principais elementos de análise e aplica entrevistas aos participantes da rede para compreender as dinâmicas do grupo. Zattar (2017) descreve as redes instituídas como complexas, de caráter inter e transdisciplinar, em que é necessária a participação integrada de diversos pesquisadores para reflexão e desenvolvimento de ações junto à sociedade e na produção de conhecimento científico para o campo. Conclui que mesmo diante de tamanha variedade de conhecimentos produzidos, as fronteiras disciplinares precisam ser extrapoladas como alternativa.

\section{CONSIDERAÇÕES FINAIS}

As redes de conhecimento para Latour $(2000,2006,2017)$ se formam da junção e movimento de distintas características: informações, inscrições, fenômenos, objetos de estudo, por meio de translações impulsionadas por mediadores/atores em espaços físicos e abstratos, não formais e formais, sendo bibliotecas, laboratórios e instituições de ensino e pesquisa ambientes primordiais para impulsão das redes. As pesquisas acima descritas certificam esta variabilidade e multiplicidade.

A partir dos estudos analisados, as redes de conhecimento abordam a produção do conhecimento científico em universidades, centros de pesquisa, grupos de estudos e repositórios científicos. Referem-se a diferentes objetos, seja na esfera de concepção, com base em grupos de estudos e linhas de pesquisa, seja na esfera da documentação, com análises sobre artigos, bases de dados e periódicos científicos ou, em ambos, por meio dos diretórios de pesquisa.

É necessário compreender e registrar a dinâmica das redes de conhecimento dos espaços formais de pesquisa, pois mesmo parecendo tão clara sua relevância científica, social e econômica, estes locais têm sofrido frequentes ataques (dentro do campo de lutas simbólicas) com o intuito de desestabilizar e fragilizar sua importância diante da sociedade. As universidades públicas correm risco de comprometer a qualidade dos serviços prestados à sociedade por estarem suscetíveis a políticas de governo e políticos que buscam sua precarização. 


\section{REFERÊNCIAS}

Alvarenga, G. C. d. L. P. d. (2009). Redes de co-autoria científica no programa de pós-graduação em geociências da ufrgs. (Dissertação de mestrado). Universidade Federal do Rio Grande do Sul, Brasil.

Alvarenga, G. C. d. L. P. d. (2012). Produção do conhecimento científico brasileiro: o caso de uma instituição de pesquisas em campinas. (Tese de doutorado). Universidade Estadual de Campinas, Brasil.

Alvarenga, G. C. d. L. P. d. (2013). Capital social e humano e a produção cientifica: um estudo sobre redes sociais de conhecimento em uma universidade pública brasileira. (Tese de doutorado). Universidade Federal da Bahia, Brasil.

Alvarenga, G. C. d. L. P. d. (2014). Diretório dos grupos de pesquisa do cnpq - como ferramenta de análise de redes: um estudo de caso do cds/unb. (Dissertação de mestrado). Universidade de Brasília, Brasil.

Bardin, L. (2009). Análise de conteúdo. Lisboa: Edições 70 .

Bauer, M. (2013). Análise de conteúdo clássica: uma revisão. In M. Bauer \& G. Gaskel (Eds.), Pesquisa qualitativa com texto imagem e som: um manual prático (11a. ed.). Petrópolis: Vozes.

Capurro, R. (2003). Epistemología y ciencia de la información. Texto apresentado em confeência. Recuperado de https://fakenews.publicdatalab.org.

Capurro, R., \& Hjørland, B. (2007). O conceito de informação. Perspectivas em Ciência da Informação, 12(1), 148-207. Recuperado de http://www.scielo.br/scielo.php?script=sci _arttext\&pid=S1413-99362007000100012\&lang=pt.

Caribé, R. d. C. d. V. (2015). Comunicação científica: reflexões sobre o conceito. Informação e Sociedade, $25(3)$, 01-16. Recuperado de http://www.periodicos.ufpb.br/ojs/ index.php/ies/article/view/23109/14530.

Costa, V. d. S. (2011). A pesquisa nos grupos de pesquisa: cibernética pedagógica - laboratório de linguagens digitais lld. (Dissertação de mestrado). Universidade de São Paulo, Brasil.

Costa, V. d. S. (2016). Rede de pesquisa no instituto de pesquisa económica aplicada (ipea): uma análise da produção científica a partir dos textos para discussão (td). (Dissertação de mestrado). Universidade de Brasília, Brasil.

Gil, A. C. (2008). Métodos e técnicas de pesquisa social. (18a. ed.). São Paulo: Atlas.

González de Gómez, M. N. (2012). As ciências sociais e a questão da informação. Morpheus: Revista Eletrônica de Ciências Humanas, 9(14), 18-37. Recuperado de http://www4.unirio.br/morpheusonline/numero14-2012/ artigos/nelida_pt.pdf.

Instituto Brasileiro de Informação em Ciência e Tecnologia (IBICT). ([2018]). Biblioteca brasileira de teses e dissertações: sobre a bbtd. Rio de Janeiro: IBICT. Recuperado de http://bdtd.ibict.br/vufind/Content/history.

Kuhn, T. (2007). A estrutura das revoluções cientificas. São Paulo: Perspectiva.

Latour, B. (2000). Ci encia em ação: como seguir cientistas e engenheiros sociedade afora. São Paulo: Editora da UNESp.

Latour, B. (2006). Redes que a razão desconhece. In M. Baratin \& C. Jacob (Eds.), O poder das bibliotecas (2a. ed., p. 21-44). Rio de Janeiro: Editora da UERJ.

Latour, B. (2017). A esperança de pandora: ensaios sobre a realidade dos estudos cient íficos. São Paulo: Editora da UNESp.

Martins, G. d. A., \& Theóphilo, C. R. (2007). Metodologia da investigação cient ífica para ciências sociais aplicadas. São Paulo: Atlas.

Mc Garry, K. (1999). O contexto dinâmico da informação: uma análise introdutória. Brasília: Briquet de Lemos.

Minayo, M. C. d. S. (2001). Pesquisa social. teoria, método e criatividade. (18a. ed.). Petrópolis: Vozes.

Zattar, M. (2017). Prática informacional em redes no domínio da governança da água: um estudo sobre o processo de produção do conhecimento. (Tese de doutorado). Universidade Federal do Rio de Janeiro, Brasil.
Stueber, K. \& Teixeira, M. R. F. (2019). Redes de conhecimento na comunicação científica em âmbito formal: panorama via Biblioteca Brasileira de Teses e Dissertações (2008-2018). AtoZ: novas práticas em informação e conhecimento, 8(2), 91 - 97. Recuperado de: http://dx.doi.org/10.5380/atoz.v8i2.72923 